\title{
Study on Unmanned Hybrid Unmanned Surface Vehicle and Unmanned Underwater Vehicle System
}

\author{
Han-Sol Jin®1, Hyunjoon Cho®1, Ji-Hyeong Lee®1, Huang Jiafeng®1, \\ Myung-Jun Kim ${ }^{\oplus 1}$, Ji-Youn $\mathrm{Oh}^{2}$ and Hyeung-Sik Choi ${ }^{3}$ \\ ${ }^{1}$ Graduate Student, Department of Mechanical Engineering, Korea Maritime and Ocean University, Busan, Korea \\ ${ }^{2}$ Post Doctor, Maritime ICT RED Center, Korea Institute of Ocean Science E Technology, Busan, Korea \\ ${ }^{3}$ Professor, Department of Mechanical Engineering, Korea Maritime and Ocean University, Busan, Korea
}

KEY WORDS: Unmanned surface vehicle, Unmanned underwater vehicle, Autonomy, Autonomous control, Integrated system, Field test

ABSTRACT: Underwater operating platforms face difficulties regarding power supply and communications. To overcome these difficulties, this study proposes a hybrid surface and underwater vehicle (HSUV) and presents the development of the platform, control algorithms, and results of field tests. The HSUV is capable of supplying reliable power to the unmanned underwater vehicle (UUV) and obtaining data in real time by using a tether cable between the UUV and the unmanned surface vehicle (USV). The HSUV uses global positioning system (GPS) and ultra-short base line sensors to determine the relative location of the UUV. Way point (WP) and dynamic positioning (DP) algorithms were developed to enable the HSUV to perform unmanned exploration. After reaching the target point using the WP algorithm, the DP algorithm enables USV to maintain position while withstanding environmental disturbances. To ensure the navigation performance at sea, performance tests of GPS, attitude/heading reference system, and side scan sonar were conducted. Based on these results, manual operation, WP, and DP tests were conducted at sea. WP and DP test results and side scan sonar images during the sea trials are presented.

\section{Introduction}

The importance of unmanned surface vehicles (USVs) and unmanned underwater vehicles (UUVs) has recently increased as various marine resources, such as petroleum and gas, are gaining importance (Bentley, 2002; Nicholson and Healey, 2008). Although massive amounts of resources are present in the oceans, marine exploration is difficult to perform due to ocean currents and weather conditions (Hou, 2013). Particularly, the operation of a UUV in water poses difficulties that are not encountered on land, such as difficulties in supplying power, communication issues, low visibility, and water pressure. Recently, studies on platforms that combine USVs and UUVs have been performed to resolve such difficulties. Sarda and Dhanak proposed a hybrid surface and underwater vehicle (HSUV) that assists in launching and recovering vessels using tether cables (Sarda and Dhanak, 2017). However, this platform is limited in terms of power supply and real-time transmission of large amounts of data. Zwolak et al. proposed a similar platform that can additionally acquire large amounts of data (Zwolak et al., 2017); however, this platform faces difficulties in real-time data transmission and power supply for long-term operation. In addition, it is difficult to obtain the real-time location of a UUV.

To resolve the aforementioned limitations, an HSUV that combines a USV and a UUV, retaining their advantages, is proposed in this study. The USV used is a stable catamaran designed to easily accelerate (Molland et al., 1994). The UUV used is also a catamaran that receives power from the USV, liberating space that would be occupied by batteries and increasing the payload capacity. Sensor data of the UUV can be verified in real time on land through a tether cable, and control commands can be transmitted. The power supplied from the USV enables UUV to be operated for a long period of time using its function for real-time communication with land. In addition, images from the UUV can be acquired in real time on land. The real-time relative locations of the USV and UUV can be identified by utilizing ultra-short base line (USBL) sensors.

The system components, kinetics, and control algorithm of the

Received 30 June 2020, revised 12 October 2020, accepted 19 October 2020

Corresponding author Hyeung-Sik Choi: +82-51-410-4297, hchoi@kmou.ac.kr

It is a recommended paper from the proceedings of 2019 Fall Conference of the Korea Marine Robot Technology (KMRTS).

(c) 2020, The Korean Society of Ocean Engineers

This is an open access article distributed under the terms of the creative commons attribution non-commercial license (http://creativecommons.org/licenses/by-nc/4.0) which permits unrestricted non-commercial use, distribution, and reproduction in any medium, provided the original work is properly cited. 
developed HSUV are described in Sections 2, 3, and 4, respectively. Kinematic performance and control test results from offhshore tests are described in Section 5.

\section{System Structure of Hybrid Surface \& Underwater Vehicle}

Fig. 1 shows the structure of the HSUV constructed for this study. Operation console commands are transferred to the USV and UUV via a hub through wireless bridges, and USV and UUV are connected through a tether cable. The total weight of the HSUV, excluding the operation console, is $350 \mathrm{~kg}$.

\subsection{System Components of the Hybrid Surface and Underwater Vehicle}

The system of the HSUV was constructed as in Fig. 2, and the console was constructed to control it. A personal computer (PC) in a land station has a program written in $\mathrm{C \#}$ to identify the real-time condition of the surface and underwater vehicles via a graphical user interface (GUI). The main controller of the USV uses CYBCKIT-059 PSoC to operate the propellers, and the code was written to autonomously sail based on global positioning system (GPS) and attitude and heading reference system values. The surface vehicle is equipped with two cameras, a battery management system (BMS), a USBL, and light detection and ranging (Lidar), which can be verified

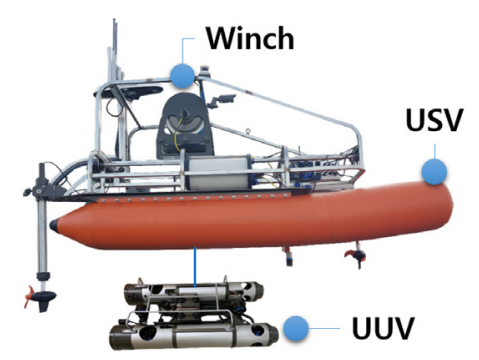

Fig. 1 HSUV components on the operation console. The main controller of the underwater vehicle uses Pixhawk, and the cameras and side scan sonar data can be verified in real time on the operation console.

\subsection{System Components of Unmanned Surface Vehicle}

The components of the USV are shown in Fig. 3. The USV is a stable catamaran that has a buoyancy gain and can quickly accelerate. It transports the UUV to an exploration point by realizing GPS location information-based autonomous sailing. Voltage decreases and efficiency are considered to install batteries of $48 \mathrm{~V}$ and $24 \mathrm{~V}$ in the USV. The winch system of the USV consists of a brushless direct-current motor, a slip ring, and a level shaft. This system stably winds the tether cable, supplies electric current to the UUV, and controls exploration depths. The lidar and camera sensors were attached to recognize obstacles. The Lidar is capable of $360^{\circ}$ omnidirectional identification. Front and docking verification cameras provide visual assistance in operation. The specifications of the USV are listed in Table 1.

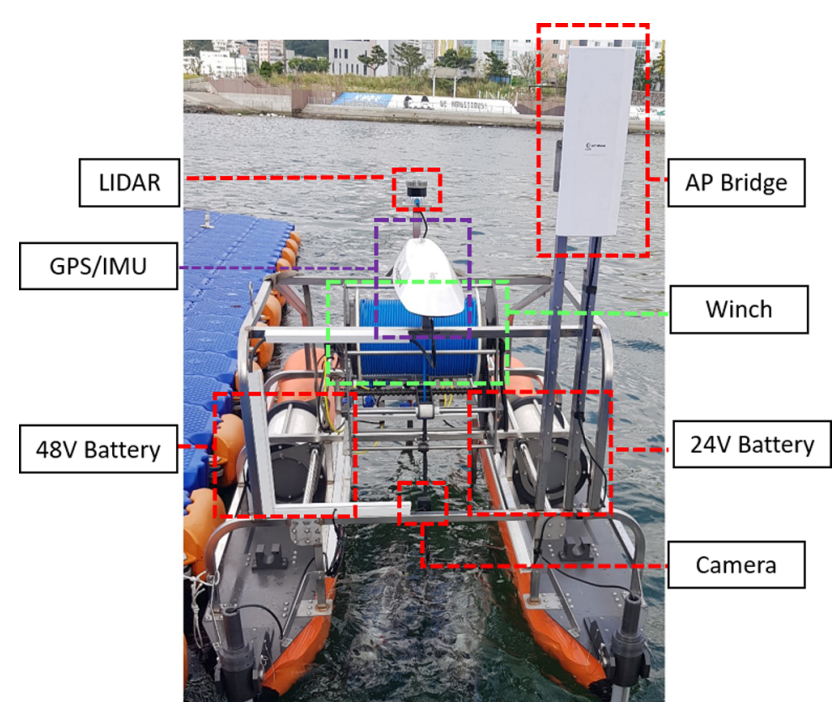

Fig. 3 USV components

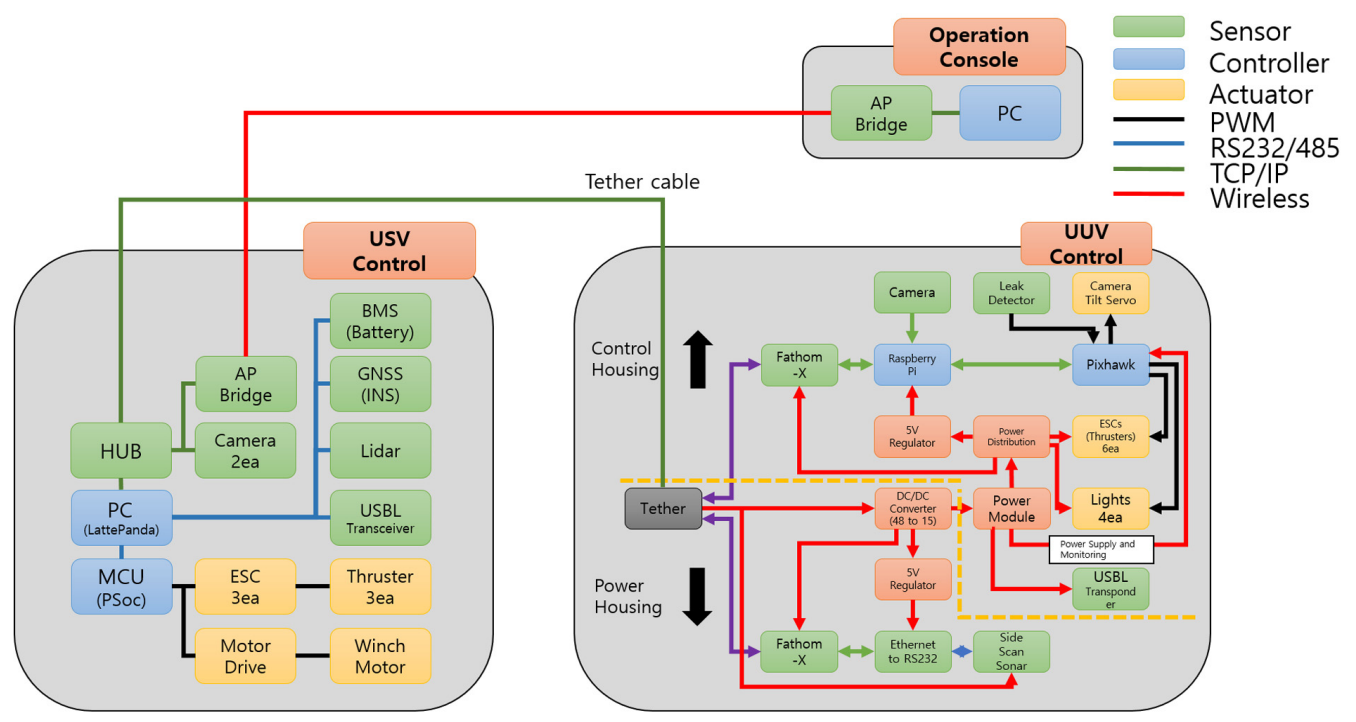

Fig. 2 HSUV system configuration 
Table 1 USV and UUV specifications

\begin{tabular}{cc}
\hline Item & Specification \\
\hline USV size $(\mathrm{m})$ & $3.3 \times 2.5 \times 1.6$ \\
USV weight $(\mathrm{kg})$ & 310 \\
\hline UUV size $(\mathrm{m})$ & $1.15 \times 0.55 \times 0.3$ \\
UUV weight $(\mathrm{kg})$ & 49.5 \\
\hline
\end{tabular}

\subsection{System Components of Unmanned Underwater Vehicle}

Fig. 4 shows the UUV components. The UUV has an underwater tilt camera and a side scan sonar as sensors for exploring seabed terrain and underwater structures. The UUV weighs $49.5 \mathrm{~kg}$ on land and has a negative buoyancy of $14 \mathrm{~kg}$. It is constructed in the form of a catamaran with relatively stable rolling to acquire high precision data from the side scan sonar. Depths are controlled using the depth sensor-based winch system and six propellers are used in the hull to allow six-axis control. The kinematic performance and USBL data are used to accurately identify the location of the underwater vehicle and perform various tasks. Ethernet is used for data transmission, and the data transmission rate to/from the land station is approximately 86 $\mathrm{Mb} / \mathrm{s}$. This value is verified using the iperf program, as shown in Fig. 5 , and the data of each sensor can be verified in real time at the land station. The underwater camera is mounted in the dome-shaped space on the front of the underwater vehicle and its direction can be adjusted up to $90^{\circ}$ using a tilt servo motor. It is a low-light camera for exploration in dark water and has horizontal and vertical visual fields

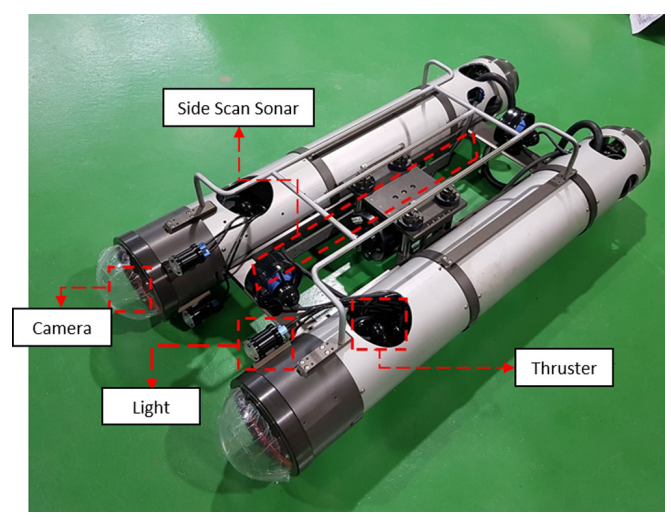

Fig. 4 UUV components

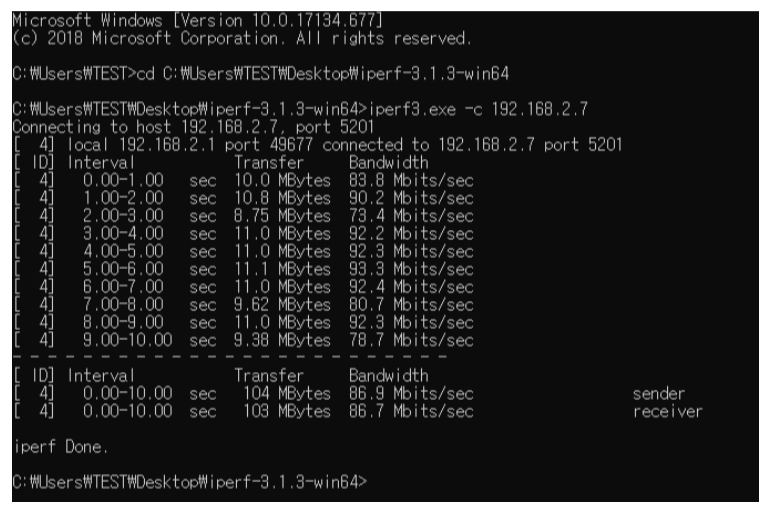

Fig. 5 Ethernet speed measurement result using 'iperf' program of $80^{\circ}$ and $68^{\circ}$, respectively.

Considering voltage decreases through the tether cable, the underwater vehicle received $48 \mathrm{~V}$ direct-current power. Data are transmitted between the surface and underwater vehicles through the tether cable via power over Ethernet. A light whose brightness can be adjusted up to $1,500 \mathrm{~lm}$ was attached for dark underwater exploration.

\subsection{Operation Console and GUI Program}

Fig. 6 shows the operation console of the HSUV. The operation console was built to verify side scan sonar data, cameras, and the current states and control commands of the USV and UUV in real time. A commercial PC, a joystick, and Wi-Fi equipment are loaded in a case, and an inverter is mounted inside the console to supply power to access point (AP) bridges. The console has sufficient space to efficiently store the power cable, mouse, and joystick required for operation. A cooling fan was also attached, considering the strong direct sunlight on the sea.

Fig. 7 shows a part of the operation program used in this study. This program was written to verify numerical data of surface and underwater vehicles and GPS and intuitively view the data via GUI. Google Map is provided to verify real-time locations and paths. Joystick, way point (WP) and dynamic positioning (DP) modes, and winch control are implemented as functions of the program. The four cameras of the HSUV are connected to the operation console via dual monitors to conveniently monitor their output.

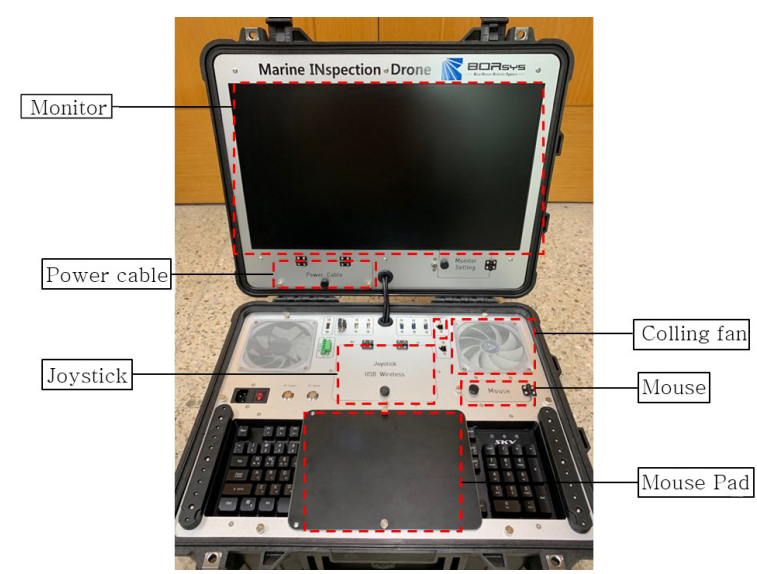

Fig. 6 Operation console components

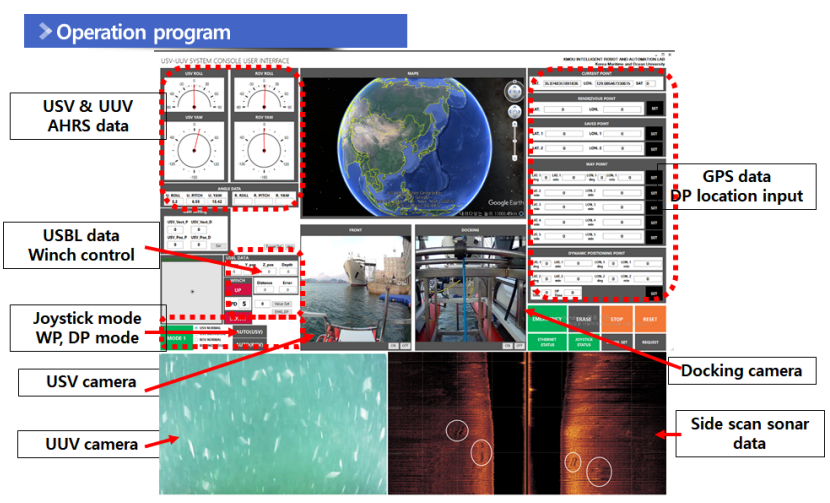

Fig. 7 Operation console program 


\section{Kinetics of Hybrid Surface and Underwater Vehicle}

The dynamics of the HSUV is based on the Newton-Euler equations and vectors. The USV moves along the $\mathrm{x}$-axis and $\mathrm{y}$-axis; hence, its motion can be expressed as surge, sway, and yaw. The notation associated with each axis is presented in Table 2. Considering the Coriolis centripetal force and attenuation acting on the USV, the dynamic equations can be expressed as Eqs. (1)-(3). The detailed definitions for the parameters and states are described in Fossen (2011) and Cho et al. (2020).

Table 2 Notation of Society of Naval Architects and Marine Engineers for USV and UUV

\begin{tabular}{|c|c|c|c|}
\hline Translational motion & Force & Position & Linear velo \\
\hline Surge & $X$ & $x$ & $u$ \\
\hline Sway & $Y$ & $y$ & $v$ \\
\hline Heave & $Z$ & $z$ & $w$ \\
\hline Rotational motion & Moment & Euler angle & Angular velc \\
\hline Roll & $K$ & $\phi$ & $p$ \\
\hline Pitch & M & $\theta$ & $q$ \\
\hline Yaw & $N$ & $\psi$ & $r$ \\
\hline \multicolumn{4}{|c|}{$\begin{array}{l}\left(m-X_{\dot{u}}\right) \dot{u}-m u r+\left(Y_{\dot{v}} v_{r} r+Y_{\dot{r}} r-m X_{G} r\right) r-\left(X_{u}+X_{u|u|}|u|\right) u \\
=\tau_{X}\end{array}$} \\
\hline \multicolumn{4}{|c|}{$\begin{array}{l}\left(m-Y_{\dot{v}}\right) \dot{v}+\left(m x_{G}-Y_{\dot{r}}\right) \dot{r}-m u r-X_{\dot{u}} u_{r} r-\left(Y_{v}+Y_{v|v|}|v|\right) v- \\
\left(Y_{r}+Y_{r|r|}|r|\right) r=\tau_{Y}\end{array}$} \\
\hline \multicolumn{4}{|c|}{$\begin{array}{l}\left(m x_{G}-N_{\dot{v}}\right) \dot{v}+\left(I_{Z}-N_{\dot{r}}\right) \dot{r}-\left(Y_{\dot{v}} v_{r} r-Y_{\dot{r}} r+m x_{G} r\right) u+X_{\dot{u}} u_{r} v \\
-\left(N_{v}+N_{v|v|}|v|\right) v-\left(N_{r}+N_{r|r|}|r|\right) r=\tau_{N}\end{array}$} \\
\hline
\end{tabular}

Here, $m$ is the mass, $I$ is the mass moment of inertia, and $\tau$ is the thrust of two rear thrusters attached to the USV.

Eqs. (4)-(6) represent the motion of the UUV. The UUV follows the considerations for USV, but surge, sway, and yaw are considered as relative locations and relative direction angles. The subscript $R$ in Eqs. (4)-(6) was used to avoid confusion with the equations of the USV. The symbol on the right side of sigma represents the sum of environmental disturbance and thrust.

$$
\begin{aligned}
& m_{R}\left[\dot{u_{R}}-v_{R} r_{R}+w_{R} q_{R}-x_{G_{R}}\left(q_{R}^{2}+r_{R}^{2}\right)+y_{G_{R}}\left(p_{R} q_{R}-\dot{r_{R}}\right)\right. \\
& +z_{G_{R}}\left(p_{R} r_{R}+\dot{q_{R}}\right)=\sum X_{R} \\
& m_{R}\left[\dot{v_{R}}-w_{R} p_{R}+u_{R} r_{R}-y_{G_{R}}\left(r_{R}^{2}+p_{R}^{2}\right)+z_{G_{R}}\left(q_{R} r_{R}-\dot{p_{R}}\right)\right. \\
& +x_{G_{R}}\left(p_{R} q_{R}+\dot{r}_{R}\right)=\sum Y_{R} \\
& I_{Z_{R}} \dot{r}+\left(I_{y_{R}}-I_{x_{R}}\right) p_{R} q_{R}+m_{R}\left[x_{G_{R}}\left(\dot{v_{R}}-w_{R} p_{R}+u_{R} r_{R}\right)\right. \\
& \left.-y_{G_{R}}\left(\dot{u}-v_{R} r_{R}+w_{r} p_{R}\right)\right]=\sum N_{R}
\end{aligned}
$$

\section{Control Algorithm of Hybrid Surface and Underwater Vehicle}

\subsection{Dynamic Positioning Algorithm}

The DP algorithm of the USV is illustrated in Fig. 8. The DP process is performed as follows: when the USV moves out of a target radius due to the effect of tidal current or wind, it obtains its rotational direction angle toward a DP point and adjusts its heading. The platform moves when the heading of the platform coincides with the target direction angle, and stops when it reaches the target point.

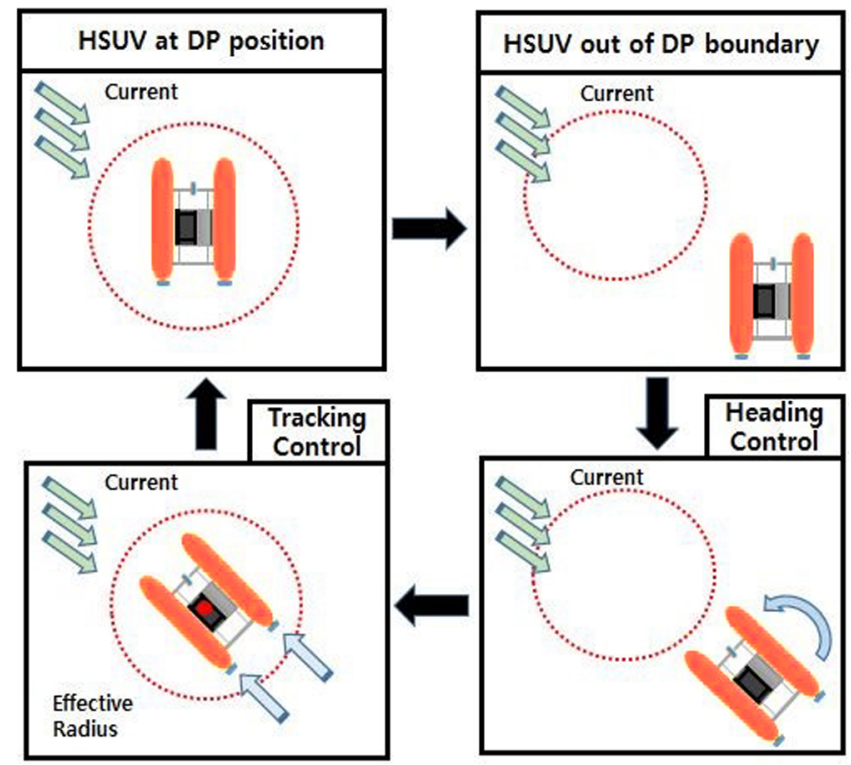

Fig. 8 DP algorithm

$$
\left[X_{k}-X(t)\right]^{2}+\left[Y_{k}-Y(t)\right]^{2}<\rho_{c}^{2}
$$

Eq. (7) represents the distance error. $[X(t), Y(t)]$ represents the location of the HSUV, represents the target point location, and $\rho_{c}$ is the target radius.

\subsection{Way Point Algorithm}

The WP algorithm is illustrated in Fig. 9. When the latitude and longitude of a target point are given, the USV obtains its target direction angle using the line of sight and adjusts its heading. The platform moves when the heading of the platform corresponds to the target direction angle, and stops when it reaches the target point. This process is completed and repeated toward the next WP.

$$
\phi_{U}=\tan ^{-1}\left[\frac{Y_{k}-Y(t)}{X_{k}-X(t)}\right]
$$

Eq. (8) represents the direction angle error. $[X(t), Y(t)]$ represents the location of the HSUV, and $\left[X_{k}, Y_{k}\right]$ represents the target point location. 


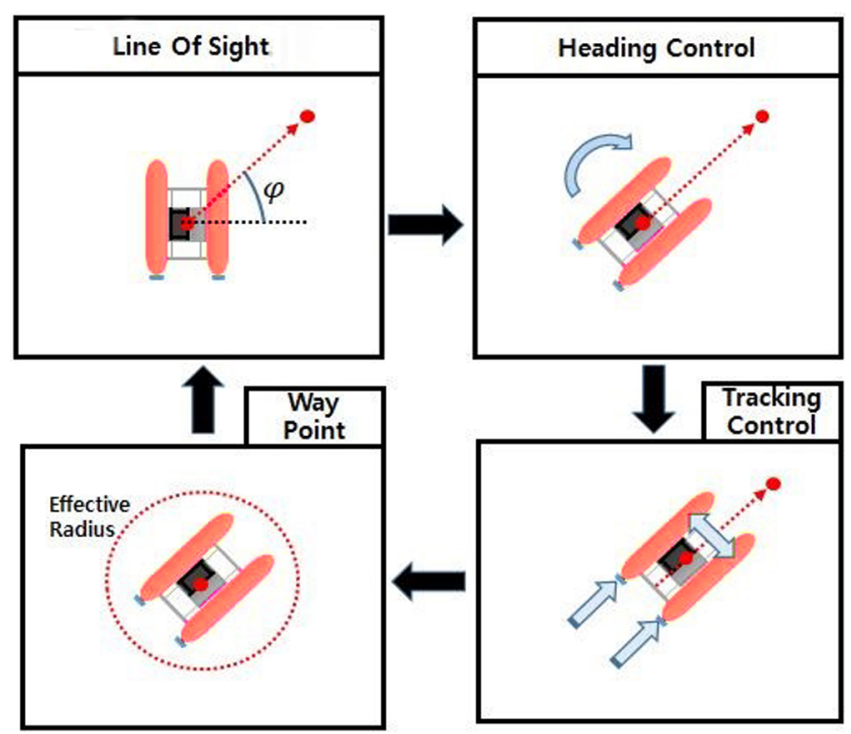

Fig. 9 WP algorithm

\section{Offshore Tests}

Offshore tests were performed on the relatively calm coast of the Korea Maritime and Ocean University to evaluate the performance of the proposed HSUV and its control system. The offshore tests include accuracy tests of the GPS, which is the key sensor on land, and communication tests. Based on these tests, route tracking tests of the USV, DP, and performance tests of the side scan sonar were performed.

Tests for the communication distance of the platform were performed prior to the main tests. The communication equipment used in the unmanned vessel has a high effective transmission rate of 360 $\mathrm{Mb} / \mathrm{s}$ and additionally uses an omnidirectional antenna.

The test results indicated that data are effectively received and transmitted up to $890 \mathrm{~m}$ in the absence of obstacles between thewireless communication devices. Fig. 10 shows a picture of the offshore tests performed.

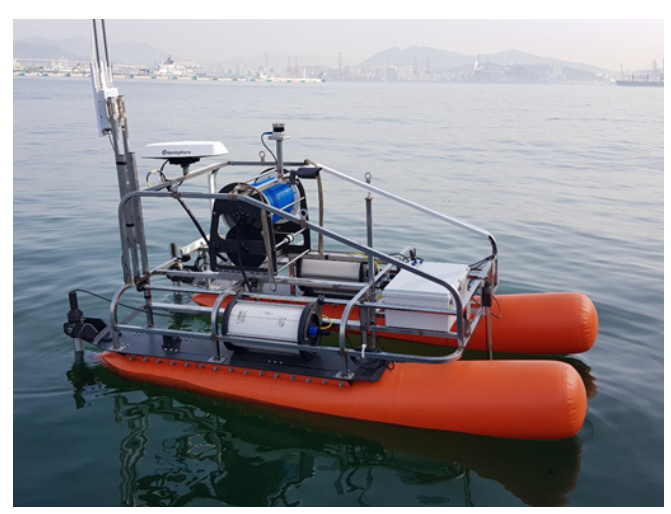

Fig. 10 Sea experiment

\subsection{GPS Sensor Tests}

The GPS sensor used in the proposed hybrid system provides true north data, magnetic north data, and altitude values. Tests for moving

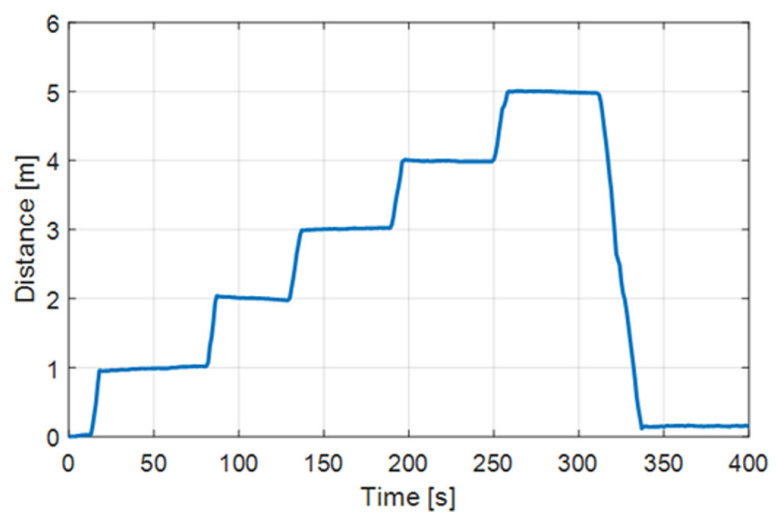

Fig. 11 GPS performance test

the system $5 \mathrm{~m}$ and then returning to the initial point were performed to verify the reliability of sensor values. The test results are shown in Fig. 11.

\subsection{WP Tests}

Route control tests were conducted on the USV to individually evaluate the accuracy of the sensors.

The WP algorithm of Section 4.2 was used for the USV to reach four WPs on the sea in these tests. Fig. 12 shows the corresponding results, and Table 3 lists the WPs designated in the operation console. The latitude and longitude of the four WPs were input into the operation console and WPs 1-4 were reached from the start point. The maximum error obtained for the WPs was $2.6 \mathrm{~m}$.

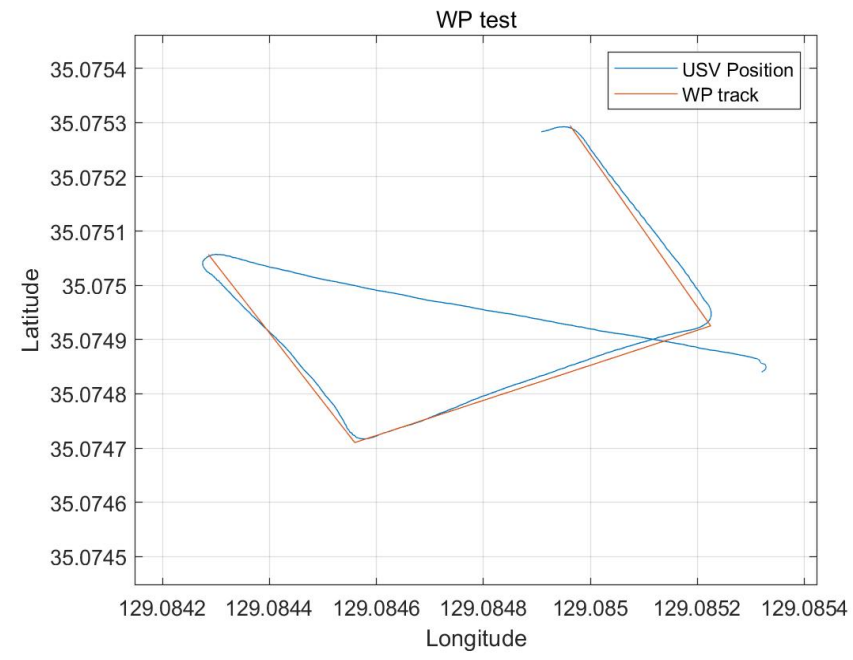

Fig. 12 WP results of USV

Table 3 USV way points

\begin{tabular}{ccc}
\hline Way point & Latitude & Longitude \\
\hline Start point & 35.0748403 & 129.0853198 \\
Way point 1 & 35.0749253 & 129.0852248 \\
Way point 2 & 35.0752943 & 129.0849620 \\
Way point 3 & 35.0750570 & 129.0842860 \\
Way point 4 & 35.0747102 & 129.0845596 \\
\hline
\end{tabular}




\subsection{Side Scan Sonar Performance Tests}

A side scan sonar was mounted on an underwater drone to measure the state of the bottom of a river. The tests were performed on the estuary bank of the brackish water zone of Nakdong River. As the river is not deep, the UUV was lowered $0.5 \mathrm{~m}$, towed by the USV, and moved straight-line distances. Fig. 13 shows pictures of captured bridge posts and fishes.

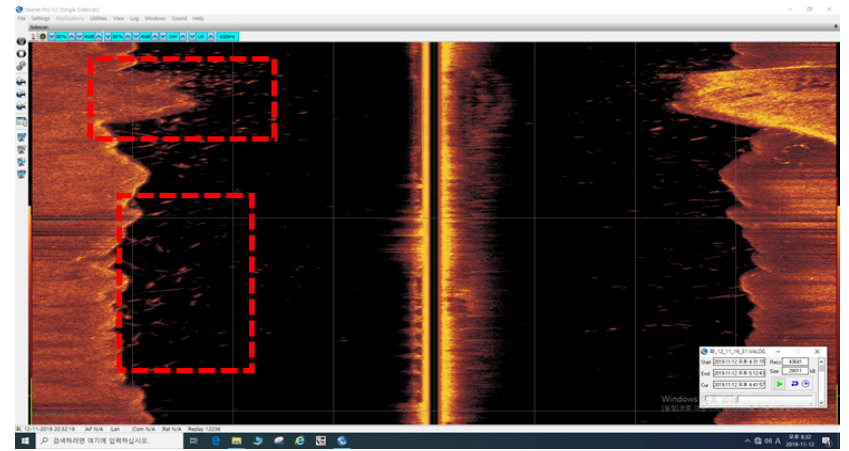

Fig. 13 Search result of the river bottom using side scan sonar

\subsection{Dynamic Positioning Tests}

The DP algorithm of Section 4.1 developed for this study was used to perform the DP of the USV on the sea. The DP algorithm was constructed to operate the controller when the USV moves out of a target radius of $3.0 \mathrm{~m}$ to extend the operation time of the USV. With the algorithm, the $3.3 \mathrm{~m}$ USV was controlled to remain within a certain radius. Fig. 14 shows the results of ten minutes of DP in measurement units of $500 \mathrm{~ms}$. The test results generally satisfied the target error radius of $3.0 \mathrm{~m}$, and the average DP error was $2.03 \mathrm{~m}$, which shows that DP was adequately performed in comparison to the full length of the vehicle.

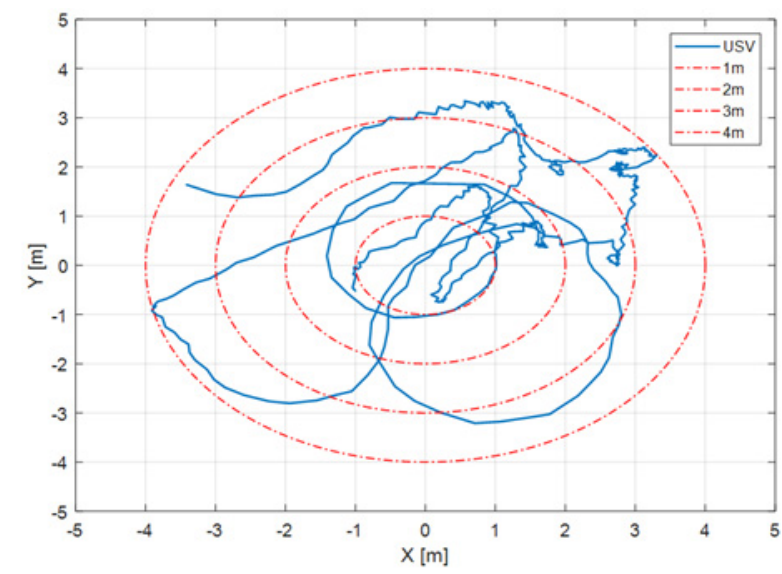

Fig. 14 DP results for the USV

\section{Conclusions}

In this study, the structure and control system of the HSUV were designed and constructed and basic performance tests were performed. The sensor data of the USV and UUV can be verified in real time through the tether cable, and the UUV can be used for a long period of time. The individual sensor performance tests were performed, followed by offshore tests using the controller algorithms constructed in Section 4 to verify the performance. The maximum error of the WPs of the USV was $2.60 \mathrm{~m}$, and the average DP error was $2.03 \mathrm{~m}$, which indicates the excellence of the control system. In addition, the side scan sonar of the UUV was used in the same sea area to explore the bottom of a river. The river, surrounding terrain, and fish search information could be monitored in real time at the land station by utilizing the real-time communication system.

\section{References}

Bentley, R.W. (2002). Global Oil \& Gas Depletion: an Overview. Energy Policy, 30(3), 189-205. https://doi.org/10.1016/S03014215(01)00144-6

Cho, H., Jeong, S.-K., Ji, D.-H., Tran, N.-H., Vu, M.T., \& Choi, H.-S., (2020). Study on Control System of Integrated Unmanned Surface Vehicle and Underwater Vehicle. Sensors, 20(9), 2633. https://doi.org/10.3390/s20092633

Fossen, T.I. (2011). Handbook of Marine Craft Hydrodynamics and Motion Control ( $1^{\text {st }}$ ed.). New Jersey, USA: Jhon Wiley \& Sons.

Hou, W. (2013). Ocean Sensing and Monitoring: Optics and Other Methods ( $1^{\text {st }}$ ed.). Washington, USA: SPIE. https://doi.org/ $10.1117 / 3.1002079$

Nicholson, J.W., \& Healey, A.J. (2008). The Present State of Autonomous Underwater Vegicle (AUV) Applications and Technologies. Marine Technology Society Journal, 42(1), 44-51. https://doi.org/10.4031/002533208786861272

Molland, A.F., Wellicome, J.F., \& Couser, P.R. (1994). Resistance Experiments on a Systematic Series of High Speed Displacement Catamaran Forms: Variation of Length-displacement Ratio and Breadth-draught Ratio (Ship Science Reports, 71). Southampton, UK: University of Southampton.

Sarda, E.I., \& Dhanak, M.R. (2017). A USV-Based Automated Launch and Recovery System for AUVs. IEEE Journal of Oceanic Engineering, 42(1), 37-55. https://doi.org/10.1109/JOE.2016. 2554679

Zwolak, K., Simpson, B., Anderon, R., Bazhenowa, E., Falconer, R., Kearns, T., ... \& Tinmouth, N. (2017). An Unmanned Seafloor Mapping System: The Concept of an AUV Integrated with the Newly Designed USV SEA-KIT. Proceedings of the OCEANS, Aberdeen, UK. https://doi.org/10.1109/OCEANSE.2017.8084899

\section{Author ORCIDs}

$\begin{array}{ll}\text { Author name } & \text { ORCID } \\ \text { Jin, Han-Sol } & 0000-0002-3400-3272 \\ \text { Cho, Hyunjoon } & 0000-0003-1084-8542 \\ \text { Lee, Ji-Hyeong } & 0000-0002-3226-6036 \\ \text { Huang, Jiafeng } & 0000-0002-2866-0778 \\ \text { Kim, Myung-Jun } & 0000-0002-9018-3837 \\ \text { Oh, Ji-Youn } & 0000-0003-3588-5892 \\ \text { Choi, Hyeung-Sik } & 0000-0002-4060-8163\end{array}$

